\title{
A Geometric Property of the Laplacian matrix of a Connected Nonsingular Mixed Graph
}

\author{
Zheng-Da Zhou and Shi-Cai Gong \\ School of Science, Zhejiang University of Science and Technology, Hangzhou 310023, China \\ Correspondence should be addressed to Shi-Cai Gong; scgong@zafu.edu.cn
}

Received 5 August 2019; Accepted 29 October 2019; Published 28 February 2020

Guest Editor: Jia-Bao Liu

Copyright (C) 2020 Zheng-Da Zhou and Shi-Cai Gong. This is an open access article distributed under the Creative Commons Attribution License, which permits unrestricted use, distribution, and reproduction in any medium, provided the original work is properly cited.

In this paper, we give a geometric interpretation of the Laplacian matrix of a connected nonsingular mixed graph which generalizes the results of M. Fiedler (M. Fiedler, Geometry of the Laplacian, Linear Algebra Appl., 2005, 403: 409-413). In addition, the relations of geometric properties between a connected (singular or nonsingular) mixed graph, and all its resigned graphs will be characterized.

\section{Introduction}

Let $G=(V, E)$ be a mixed graph with vertex set $V=V(G)=$ $\left\{v_{1}, v_{2}, \ldots, v_{n}\right\}$ and edge set $E=E(G)=\left\{e_{1}, e_{2}, \ldots, e_{m}\right\}$, which is obtained from an undirected simple graph by orienting some (possibly none or all) of its edges such that one of its endpoint forms a head and the another one is a tail. The incidence matrix of $G$ is an $n \times m$ matrix $M=M(G)=$ $\left(m_{i j}\right)$ whose entries are defined by $m_{i j}=1$ if $e_{j}$ is an unoriented edge incident to $v_{i}$ or $e_{j}$ is an oriented edge with head $v_{i}, m_{i j}=-1$ if $e_{j}$ is an oriented edge with tail $v_{i}$, and $m_{i j}=0$ otherwise. The Laplacian matrix of $G$ is defined as $L(G)=\left(l_{i j}\right)_{n \times n}=M M^{T}$ (see [1-4]), where the matrix $M^{T}$ denotes the transpose of $M$. Obviously $L(G)$ is a Gram matrix and thus is positive semidefinite. $G$ is called singular (or nonsingular) if $L(G)$ is singular (or nonsingular).

A mixed graph is called quasi-bipartite if it does not contain a nonsingular cycle, or equivalently, it contains no cycles with an odd number of unoriented edges (see (1, Lemma 1)). The all-oriented graph obtained from the mixed graph $G$ by arbitrarily orienting every unoriented edge of $G$ (if one exists) will be denoted by $\vec{G}$, the signature matrix with 1 or -1 along its diagonal of a diagonal matrix will be denoted by $D$. A graph is called a resigned graph of $G$ if it is obtained from $G$ by resigning under the signature matrix $D$, and that the labelling of the vertices is the same as that of $G$, denoted by ${ }^{D} G$. Then each resigned graph of $G$ gives a resigning of the edges of $G$ (that is, some oriented edges of $G$ may turn to be unoriented and vice versa), and $L\left({ }^{D} G\right)=D^{T} L(G) D$. The following results are well known.

Lemma 1 (see [3], Lemma 2.2, and [5], Lemma 5). Let G be a connected mixed graph. Then, $G$ is singular if and only if $G$ is quasi-bipartite.

Theorem 1 (see [1], Theorem 4). Let G be a connected mixed graph. Then, $G$ is quasi-bipartite if and only if there exists a signature matrix $D$ such that $D^{T} L(G) D=L(\vec{G})$.

In this paper, we firstly give a geometric interpretation of the Laplacian matrix of a connected nonsingular mixed graph which generalizes the results of Fiedler [6] from undirected graphs to mixed graphs. Finally, the relations of geometric properties between a connected (singular or nonsingular) mixed graph and its resigned graphs will be characterized.

\section{Geometric Properties}

2.1. Nonsingular Mixed Graphs. Let $L=L(G)=\left(l_{i j}\right)_{n \times n}$ be the Laplacian matrix of a connected nonsingular mixed graph on $n(n \geq 3)$ vertices. Then, $\operatorname{rank}(L)=n$. By the wellknown equivalence of positive definite and Gram matrices, there exists uniquely $n$ linear independent vectors denoted 
by $u_{1}, \ldots, u_{n}$, in a Euclidean space $E_{n}$ of dimension $n$, such that the inner products of the vectors $u_{i}$ satisfy

$$
\left(u_{i}, u_{j}\right)=l_{i j} \quad \text { for all } 1 \leq i, j \leq n .
$$

Observe that the vectors $u_{i}$ and $u_{j}(i \neq j)$ are orthogonal if and only if there is no edge in $G$ between the vertices $i$ and $j$. If $(i, j) \in E(G)$, then the angle between $u_{i}$ and $u_{j}$ is obtuse if $(i, j)$ is oriented, the angle is acute otherwise.

Let $U=\left(u_{1}, \ldots, u_{n}\right) \in E_{n}$, then we have the following geometric interpretation of $L(G)$.

Theorem 2. Let $L=L(G)=\left(l_{i j}\right)_{n \times n}$ be the Laplacian matrix of a connected nonsingular mixed graph $G$ on $n(n \geq 3)$ vertices. Then, there exists a unique quadric $Q$ :

$$
x^{T} K^{-1} x=1
$$

such that

(i) $Q$ contains all the end-points of the vectors $u_{i}$, and

(ii) The tangent hyperplane at each of these end-points is parallel to the hyperplane containing the remaining $n-1$ vectors,

From the above equation, $x$ means the column vector of orthonormal coordinates in $E_{n}$ and $K=U U^{T}$.

Proof. Note that $K^{-1}=\left(U U^{T}\right)^{-1}=\left(U^{T}\right)^{-1} U^{-1}$, then $Z=U^{T} K^{-1} U=I_{n}$. Thus, all diagonal entries of $Z$ are equal to 1 , i.e.,

$$
u_{i}^{T} K^{-1} u_{i}=1, \quad i=1,2, \ldots, n,
$$

and all nondiagonal entries of $Z$ are 0 , which proves that quadric equation (2) contains all the end-points of the vectors $u_{i}$.

The equation of the tangent hyperplane at the end-points of $u_{k}$ to the quadric equation (2) is

$$
\frac{1}{2}\left(x^{T} K^{-1} u_{k}+u_{k}^{T} K^{-1} x\right)=1 .
$$

Note that $\left(x^{T} K^{-1} u_{k}\right)^{T}=u_{k}^{T} K^{-1} x$, then

$$
x^{T} K^{-1} u_{k}=1 \text {. }
$$

On the contrary, the hyperplane containing all vectors $u_{i}$ for $i \neq k$ satisfies the relation:

$$
x^{T} K^{-1} u_{k}=0,
$$

since $u_{i}^{T} K^{-1} u_{k}=(Z)_{i k}=0$ for $i \neq k$. Thus, both the hyperplanes are parallel, and equation (2) is indeed the equation of Q.

Assume there exists another quadric $Q^{\prime}$ :

$$
x^{T} B x=1,
$$

which holds properties (i) and (ii) mentioned above. Then, by property (i), we have

$$
u_{i}^{T} B u_{i}=1, \quad \text { for } i=1, \ldots, n .
$$

Thus, the equation of the tangent hyperplane at the endpoints of $u_{k}$ to the quadric equation (2) is

$$
x^{T} B u_{k}=1 \text {. }
$$

Consequently, by property (ii), the equation of the hyperplane containing all the remaining $n-1$ vectors is

$$
x^{T} B u_{k}=0 \text {, }
$$

since, for each $i$, the vector $u_{i}$ contains the origin as one endpoint.

Associating equations (3) and (6), we have

$$
U^{T} B U=I \text {. }
$$

Then, $B=K^{-1}$. Hence, the uniqueness follows.

The quadric $Q$ is an ellipsoid, usually called the Steiner circumscribed ellipsoid of the simplex with vertices in the end-points of the vector $u_{i}$.

Now, suppose that $v$ is an eigenvector of $L$ corresponding to the eigenvalue $\lambda$. We have $U^{T} U v=\lambda v$. Consequently, $U U^{T} U v=\lambda U v$. The vector $z=U v$ is thus an eigenvector of $K$ as well as $K^{-1}$ corresponding to the same $\lambda$ and is thus the direction of an axis of the quadric $Q$. Indeed, the halfline $x=\mu z, \mu \geq 0$, meets the quadric in the point in which the tangent is parallel to $x^{T} z=0$, thus orthogonal to $z$. We have thus a correspondence $\Gamma$ between the eigenvector of $L$ and the axis of $Q$. If the eigenvector corresponds to the eigenvalue $\lambda$, then the length of the corresponding half axis is $\sqrt{\lambda}$. Applying the method similar to that of Theorem 1 in [6], we have the following.

Theorem 3. The coordinates of the eigenvector $v$ of the Laplacian matrix $L$ are proportioned to the Euclidean coordinates of the points on the one-dimensional line generated by the vector $z$ (corresponding in $\Gamma$ ) obtained as orthogonal projections of the vectors $u_{i}$ on $z$.

Let us transform the original orthogonal coordinate system in $E_{n}$ into an other orthogonal coordinate system in which the axes coincide with the axes of the quadric $Q$. This will be performed algebraically as follows.

The matrix $K$ can be written as $O \Lambda O^{T}$, where $O$ is an orthogonal matrix and $\Lambda$ is a diagonal matrix of the eigenvalues of $\mathrm{K}$. Since $K^{-1}=O \Lambda^{-1} O^{T}$, equation (2) of $Q$ will be transformed into

$$
\begin{array}{r}
x^{T} O \Lambda^{-1} O^{T} x=1 \\
\text { or } y^{T} \Lambda^{-1} y=1,
\end{array}
$$

where $y=O^{T} x$ is again the column vector of orthogonal coordinates in the transformed system. Then, in the new system, the quadric $Q$ has the equation:

$$
\sum_{i=1}^{n} \frac{y_{i}^{2}}{d_{i}}=1 .
$$

Of course, the numbers $d_{i}$ are positive. They are equal to the eigenvalues of $L$. Let $d(G)=\max \left\{d_{i} \mid i=1, \ldots, n\right\}$ and $\alpha(G)=\min \left\{d_{i} \mid i=1, \ldots, n\right\}$. Then, $\sqrt{d(G)}$ (respectively, $\sqrt{\alpha(G)}$ ) is the semimajor (respectively, semiminor) axis of the quadric. On the contrary, $d(G)$ is the spectral radius of 
$L(G)$, and $\alpha(G)$ is the smallest eigenvalue of $L(G)$. Then, the radius of the externally tangent circle of $Q$ is $\sqrt{d(G)}$, and the radius of the internally tangent circle of $Q$ is $\sqrt{\alpha(G)}$.

Then, the vector $u_{i}$ transforms into the vector $w_{i}=O^{T} u_{i}$ for $i=1$ to $\mathrm{n}$. The following result seems interesting, we say as usual that an affine transformation is such a linear transformation which transforms points at infinity into points at infinity.

Theorem 4. Let $A$ be the affine transformation in $E_{n}$ which transforms the ellipsoid $Q$ into a hypersphere:

$$
y_{i}=\sqrt{d_{i}} Y_{i}
$$

Then, $A$ transforms the vector $w_{i}$ into vector $W_{i}=d_{i}^{(-1 / 2)} w_{i}$ which form an eutactic star, being all of equal length and spanning equal mutual angles.

Proof. By equation (14), we obtain the matrix form

$$
y=\Lambda^{(-1 / 2)} Y \text {, }
$$

where $\Lambda$ was defined earlier. Since $w_{i}=O^{T} u_{i}$ and $W_{i}=\Lambda^{(-1 / 2)} w_{i}$, we have for the matrix $W$ whose columns are the coordinates of the vectors $W_{i}, W=\Lambda^{(-1 / 2)} O^{T} U$. Therefore, the Gram matrix of the vectors $W_{i}$ is

$$
W^{T} W=U^{T} O \Lambda^{-1} \text { OTU }=U^{T} K^{-1} U=U^{T}\left(U U^{T}\right)^{-1} U=I_{n},
$$

since this is the matrix $Z$ mentioned above. Immediately, the last matrix is the Gram matrix of an eutactic star.

2.2. Mixed Graphs with the Same Underlying. In this section, we will characterize the relations of geometric properties between the graph $G$ (singular or nonsingular) and all its resigned graphs ${ }^{D} G$.

Denoted by $K^{-1}(G)$, the matrix of quadric corresponding to the graph $G$, by ${ }^{D} u_{i}$ the column vector of the matrix $U D$ since more than one graph is under discussion. Assume $L=L(G)=\left(l_{i j}\right)_{n \times n}$ be the Laplacian matrix of a connected mixed graph $G$ on $n$ vertices. Let $\aleph(G)$ be the set of all resigned graphs of a mixed graph $G$. Obviously, $\aleph(G)$ has the following properties:

(i) $G \in \aleph(G)$

(ii) All elements of $\aleph(G)$ have the same singularity as that of the graph $G$

(iii) All elements of $\aleph(G)$ can be considered as the resigned graphs of a (arbitrary) element of $\aleph(G)$

By Lemma 1 and Theorem 1, if $G$ is nonsingular, we have $L\left({ }^{D} G\right)=D^{T} L(G) D=D^{T} U^{T} U D=(U D)^{T} U D$.

Thus,

$$
K^{-1}\left({ }^{D} G\right)=\left(U D(U D)^{T}\right)^{-1}=\left(U U^{T}\right)^{-1}=K^{-1}(G),
$$

where $U=\left(u_{1}, \ldots, u_{n}\right)$ has been defined above.

Consequently, we have the following.
Theorem 5. Let $G$ be a connected nonsingular mixed graph on $n$ vertices, and let $\aleph(G)$ be the set of all its resigned graphs. Then, there exists a unique quadric Q:

$$
x^{T} K^{-1}(G) x=1,
$$

such that, for all ${ }^{D} G \in \aleph(G)$, the quadric $Q$ contains all the endpoints of the vectors ${ }^{D} u_{i}$ of ${ }^{D} G$, and the tangent hyperplane at each of these endpoints is parallel to the hyperplane containing the remaining $n-1$ vectors, where $K^{-1}(G)=$ $U U^{T} \in E_{n}$ has been defined above.

Similarly, by the result of [6], we have the following.

Theorem 6. Let $G$ be a connected singular mixed graph on $n$ vertices, and let $\aleph(G)$ be the set of all its resigned graphs. Then, there exists a unique quadric Q:

$$
x^{T} K^{-1}(G) x=\frac{n-1}{n},
$$

such that, for all ${ }^{D} G \in \aleph(G)$, the quadric $Q$ contains all the endpoints of the vectors ${ }^{D} u_{i}$ of ${ }^{D} G$, and the tangent hyperplane at each of these endpoints is parallel to the hyperplane containing the endpoints of the remaining $n-1$ vectors, where $K^{-1}(G)=U U^{T} \in E_{n-1}, U=\left(u_{1}, u_{2}, \ldots, u_{n}\right)$ which holds that $\sum_{i=1}^{n} u_{i}=0$ and $U^{T} U=L\left(G^{\prime}\right)$ the Laplacian matrix of the undirected graph $G^{\prime}$ with the same underlying graph as that of $G$.

Proof. Let $G$ be a connected singular mixed graph, then $\vec{G} \in \mathcal{N}(G)$. By Lemma 1 and Theorem 1 and by the definition of the Laplacian matrix, the matrix $L(\vec{G})$ is the same as that of the undirected graph $G^{\prime}$ with the same underlying graph. To graph $G^{\prime}$, by [6], there exists $n$ vectors, $u_{1}, \ldots, u_{n}$, in a Euclidean space $E_{n-1}$ of dimension $n-1$, such that

$$
\begin{gathered}
\sum_{i=1}^{n} u_{i}=0, \\
L\left(G^{\prime}\right)=U^{T} U,
\end{gathered}
$$

where $U=\left(u_{1}, \ldots, u_{n}\right)$

And by [6], there exists a unique quadric $Q$ :

$$
x^{T} K^{-1}\left(G^{\prime}\right) x-\frac{n-1}{n}=0
$$

such that the quadric $Q$ contains all the endpoints of the vectors $u_{i}$, and the tangent hyperplane at each of these endpoints is parallel to the hyperplane containing the endpoints of the remaining $n-1$ vectors, where $K^{-1}\left(G^{\prime}\right)=U U^{T} \in E_{n-1}$.

Let ${ }^{D} G$ be a arbitrary element of $\aleph(G)$; now, we discuss the relation of the matrices between $K^{-1}\left({ }^{D} G\right)$ and $K^{-1}\left(G^{\prime}\right)$. By Lemma 1 and Theorem 1, there exists a signature matrix $D$ such that

$$
L\left({ }^{D} G\right)=D^{T} L\left(G^{\prime}\right) D=D^{T} U^{T} U D=(U D)^{T} U D .
$$

Thus, 


$$
K^{-1}\left({ }^{D} G\right)=\left(U D(U D)^{T}\right)^{-1}=\left(U U^{T}\right)^{-1}=K^{-1}\left(G^{\prime}\right)
$$

The result follows immediately.

\section{Data Availability}

The data used to support this study are available from the corresponding author upon request.

\section{Conflicts of Interest}

The authors declare no conflicts of interest.

\section{Authors' Contributions}

S. C. Gong provided the idea of the paper and the sketchy proof of theorems; Z. D. Zhou wrote the paper.

\section{Acknowledgments}

This work was supported by the National Natural Science Foundation of China (no. 11571315) and Zhejiang Provincial Natural Science Foundation of China (no. LY20A010005).

\section{References}

[1] R. B. Bapat, J. W. Grossman, and D. M. Kulkarni, "Generalized matrix tree theorem for mixed graphs," Linear and Multilinear Algebra, vol. 46, no. 4, pp. 299-312, 1999.

[2] R. Merris, "Laplacian matrices of graphs: a survey," Linear Algebra and Its Applications, vol. 197-198, pp. 143-176, 1994.

[3] X.-D. Zhang and J.-S. Li, "The Laplacian spectrum of a mixed graph," Linear Algebra and Its Applications, vol. 353, no. 1-3, pp. 11-20, 2002.

[4] X.-D. Zhang and R. Luo, "The Laplacian eigenvalues of mixed graphs," Linear Algebra and Its Applications, vol. 362, pp. 109-119, 2003.

[5] Y. Fan and J. Li, "On graphs with small number of Laplacian eigenvalues greater than two," Linear Algebra and Its Applications, vol. 360, pp. 207-213, 2003.

[6] M. Fiedler, "Geometry of the Laplacian," Linear Algebra and Its Applications, vol. 403, pp. 409-413, 2005. 